ARTICLE

https://doi.org/10.1038/s41467-019-13795-x

\title{
Epidermal control of axonal attachment via $\beta$-spectrin and the GTPase-activating protein TBC-10 prevents axonal degeneration
}

\author{
Sean Coakley (1) ${ }^{1}$, Fiona K. Ritchie (10 ${ }^{1}$, Kate M. Galbraith ${ }^{1} \&$ Massimo A. Hilliard ${ }^{1 \star}$
}

Neurons are subjected to strain due to body movement and their location within organs and tissues. However, how they withstand these forces over the lifetime of an organism is still poorly understood. Here, focusing on touch receptor neuron-epidermis interactions using Caenorhabditis elegans as a model system, we show that UNC-70/ $\beta$-spectrin and TBC-10, a conserved GTPase-activating protein, function non-cell-autonomously within the epidermis to dynamically maintain attachment of the axon. We reveal that, in response to strain, UNC$70 / \beta$-spectrin and TBC-10 stabilize trans-epidermal hemidesmosome attachment structures which otherwise become lost, causing axonal breakage and degeneration. Furthermore, we show that TBC-10 regulates axonal attachment and maintenance by inactivating RAB-35, and reveal functional conservation of these molecules with their vertebrate orthologs. Finally, we demonstrate that $\beta$-spectrin functions in this context non-cell-autonomously. We propose a model in which mechanically resistant epidermal attachment structures are maintained by UNC-70/ $\beta$-spectrin and TBC-10 during movement, preventing axonal detachment and degeneration.

\footnotetext{
${ }^{1}$ Clem Jones Centre for Ageing Dementia Research, Queensland Brain Institute, The University of Queensland, Brisbane, QLD 4072, Australia. *email: m.hilliard@uq.edu.au
} 
F ailure to maintain the integrity of the axon, the longest and most susceptible compartment of a neuron, results in compromised neuronal function, which is a characteristic of both traumatic injury and many neurodegenerative diseases ${ }^{1}$. Axons are subject to continuous strain, mostly due to body movement and their location within skin, muscles, organs, and joints. Excessive mechanical strain, or shear stress due to external or internal traumas, can trigger degeneration of the axon. Virtually every neuron, including those of the central nervous system, is susceptible to different types of strain insults 2,3 , and mechanical strain has been implicated in the progression of neurodegenerative disease $\mathrm{e}^{4,5}$. Therefore, it is essential to understand the molecular mechanisms that protect against motioninduced injury in order to ensure that neurons and their axons maintain their correct structure and function throughout life.

The cytoskeletal spectrin-network is thought to mediate strain resistance in axons by maintaining tension via the actin-spectrin cytoskeleton $^{6,7}$. Human mutations in $\beta$-spectrin are associated with spinocerebellar ataxia, a neurodegenerative disorder that is characterized by uncoordinated gait, limb, and eye movement defects, slurred speech and swallowing difficulties ${ }^{8}$. In the nematode $C$. elegans, mutations in unc-70/ $\beta$-spectrin cause motor and sensory axons to buckle and deform during body movements, leading to spontaneous axonal breakage $e^{6,9,10}$. In particular, specialized touch receptor neurons require a functional spectrinnetwork to maintain tension within the axon in order to transduce mechanical force, and limit buckling of the axon during normal body movement ${ }^{6,7}$. During normal development, the axons of the lateral touch receptor neurons ALM and PLM become ensheathed within the epidermis (Fig. 1a, b), and are mechanically coupled to this tissue via epithelial attachment structures along the axon, similar to invertebrate and vertebrate hemidesmosomes ${ }^{11,12}$. Proper attachment between the PLM neuron and surrounding epithelium is required for both the development and maintenance of touch receptor neurons ${ }^{11,13}$. Here, we identify a function for UNC-70/ $\beta$-spectrin, the RabGTPase RAB-35, and its GTPase-activating protein (GAP) TBC10 , in preserving the axonal structure of $C$. elegans touch receptor neurons. We provide evidence that all three molecules function non-cell-autonomously within the surrounding epidermis to stablize hemidesmosomes against mechanical strain, and preserve the integrity of the ensheathed PLM axon by maintaining normal axon-epidermal attachment during movement. We show that the loss of hemidesmosomes in localized regions, and detachment of the axon from the epidermis in $t b c-10$; unc-70 mutants, is followed by breakage and subsequent degeneration of the axon. Finally, we reveal a high level of functional conservation with the human orthologs of TBC-10 and RAB-35. Our work establishes a previously unknown, non-cell-autonomous and synergistic function of $\beta$-spectrin, $\mathrm{RAB}-35$ and $\mathrm{TBC}-10$ in dynamically regulating axonal maintenance by maintaining mechanically resistant hemidesmosomes and axon-epidermal attachment. Our results highlight a direct molecular link between neurons and their surrounding tissue that is critical for axonal maintenance, but dispensable for development.

\section{Results}

TBC-10 protects against spectrin-induced damage. $C$. elegans unc-70/ $\beta$-spectrin is required to maintain neuronal integrity and to resist mechanical strain $6,7,9,10$. The spectrin-network functions both cell-autonomously within the PLM neuron, and non-cellautonomously in the surrounding epidermis to limit buckling due to compressive forces ${ }^{6}$. Animals carrying a semi-dominant mutation in the $\alpha \beta$-tetramization domain of UNC-70/ $\beta$-spectrin, unc-70(n493), present with largely normal PLM neurons
(Fig. 1c), but display a very low penetrance of axonal breakage not present in wild-type animals (Fig. 1e). To identify genes that protect against mechanically induced axonal damage, we performed an unbiased forward genetic screen using unc-70 mutants with a low penetrance of axonal damage as a sensitized background (see methods for details). From this screen, we identified a mutant allele of the highly conserved gene $t b c-10$, an ortholog of human $t b c 1 d 10 a-c$, which encodes a Rab-GAP ${ }^{14,15}$. tbc-10(vd31) mutants exhibited a dramatic increase in axonal breakage of the PLM neurons in the unc-70(n493) mutant background (Fig. 1d, e). Two additional deletion alleles of $t b c-10$ ( $t$ m2790 and tm2907) both increased the rate of PLM axonal breakage more than fivefold compared to unc-70 mutants (Fig. 1e). This enhancement of axonal breakage in the $t b c-10 ; u n c-70$ double mutants was rescued in transgenic animals carrying the wild-type $t b c-10$ genomic locus with its upstream regulatory region (Fig. 1f). In a wild-type background, $t b c-10$ mutant animals presented no spontaneous axonal breakage defects (Fig. 1h). Taken together these data demonstrate that TBC-10 functions to limit axonal damage in an unc-70-induced injury model.

TBC-10 and UNC-70/ $\beta$-spectrin function in the epidermis. To determine where $t b c-10$ is expressed, we used a cytosolic GFP reporter under the control of a $\sim 5 \mathrm{~kb}$ endogenous $t b c-10$ regulatory region for visualization. We observed GFP expression within neurons, including PLM neurons, as well as in the epidermis, intestine, and excretory canal (Supplementary Fig. 1A-D). To identify where TBC-10 acts to mediate its protective effect, we generated transgenic strains carrying a wild-type copy of $t b c-10$ driven by tissue-specific promoters. Tissue-specific expression of wild-type $t b c-10$ in either PLM neurons or muscles, was not able to rescue the increase in axonal breakage of $t b c-10$; unc-70 double mutants (Fig. 1f). In contrast, expression of wildtype $t b c-10$ under an epidermal-specific promoter fully rescued the axonal breakage phenotype (Fig. 1f), revealing a key role for TBC-10 within this tissue. To test if the function of $t b c-10$ in maintaining PLM axonal integrity is conserved across species, we expressed a complementary DNA (cDNA) encoding each of the three human orthologs, $t b c 1 d 10 a, t b c 1 d 10 b$ and $t b c 1 d 10 c$, tissuespecifically in the epidermis of $t b c-10 ; u n c-70$ double-mutant animals (Fig. 1g). Remarkably, human tbc1d10a and tbc1d10b were able to rescue the axonal breakage phenotype of the double mutants (Fig. 1g), revealing a highly conserved function of these molecules throughout evolution. $t b c-10$ shares more sequence homology with $t b c 1 d 10 a(38 \%)$ and $t b c 1 d 10 b$ (40\%), whereas $t b c 1 d 10 c(30 \%)$ is less similar. Moreover, within the critical TBCdomain $t b c 1 d 10 a$ and $t b c 1 d 10 b$ share $81 \%$ sequence identity with each other compared to $60 \%$ with $t b c 1 d 10 c^{16}$. Taken together these results demonstrate that TBC-10 functions within the epidermis to protect the axons of PLM neurons from damage caused by disruption to UNC-70/ $\beta$-spectrin, and shares functional conservation with its human orthologs TBC1D10A and TBC1D10B.

To determine whether the axonal protective effect of TBC-10 is specific for spectrin-induced injuries or more generally protects from damaging factors, we challenged $t b c-10$ single-mutant animals with other insults that cause axonal injury. Firstly, we found that compared to wild-type animals, $t b c-10$ mutants did not present with an increase in the rate of axonal degeneration in severed axon segments following laser-induced axotomy (Supplementary Fig. 2A). Furthermore, tbc-10 mutants did not display increased axonal degeneration after alteration of microtubule stability in animals lacking the a-tubulin acetyltransferase MEC17/aTAT1 (Supplementary Fig. 2B), or after treatment with colchicine or paclitaxel to destabilize and stabilize microtubules, respectively (Supplementary Fig. $2 \mathrm{C})^{17}$. These results suggest that 
a b
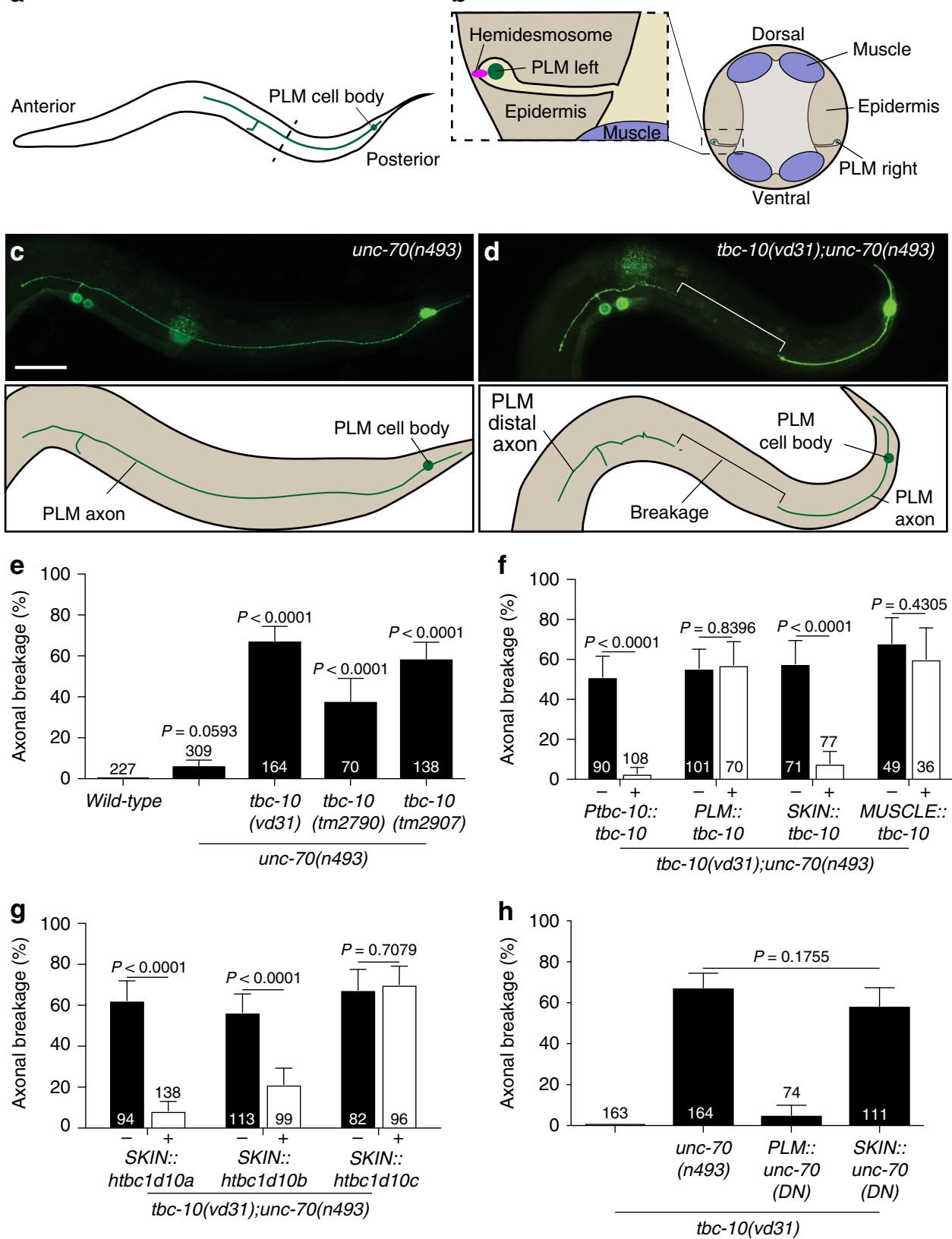

Fig. 1 TBC-10 and UNC-70/ק-spectrin function in the epidermis to protect against axonal breakage. a Scheme showing the location and morphology of the PLM touch receptor neuron. $\mathbf{b}$ A schematic transverse section through the animal (dotted line in $\mathbf{a}$ ) illustrating the region in which the PLM axon is ensheathed in the epidermis and attached via hemidesmosomes. c An image and scheme showing a typical unbroken axon of the PLM neuron in unc-70 (n493) mutants visualized with GFP (transgene zdls5(Pmec-4::GFP)). d An image and scheme showing a typical broken axon of the PLM neuron in tbc-10 (vd31); unc-70(n493) animals. e Mean penetrance of axonal breakage in wild-type, unc-70(n493) mutants, and multiple alleles of tbc-10 in an unc-70 background. $\mathbf{f}$ Mean penetrance of axonal breakage in $t b c-10$;unc-70 mutants carrying transgenes expressing wild-type tbc-10 under either an endogenous promoter, a touch receptor neuron-promoter, an epidermal-specific promoter, or a muscle-specific promoter, versus their respective non-transgenic siblings. $\mathbf{g}$ Mean penetrance of axonal breakage in tbc-10(vd31);unc-70(n493) mutants carrying transgenes expressing the wild-type human orthologs tbc1d10a-c under an epidermal-specific promoter versus non-transgenic siblings. $\mathbf{h}$ Mean penetrance of axonal breaks in tbc-10 single and tbc-10( $v d 31$ ); unc70 (n493) double mutants, versus tbc-10(vd31) mutants expressing single-copy insertions of the semi-dominant UNC-70(DN)::mKate2 driven by touch receptor neuron or epidermal-specific promoters. Scale bar in $\mathbf{c}=50 \mu \mathrm{m}$. P-values in $\mathbf{e}$ and $\mathbf{h}$ are determined from a one-way ANOVA with a Tukey multiple-comparison of proportions using an Agresti-Coull interval. $P$-values in $\mathbf{f}$ and $\mathbf{g}$ are determined from a comparison of proportions using an Agresti-Coull interval. Error bars indicate a 95\% confidence interval. $\mathrm{N}$-values are indicated on bar graphs and represent the number of individual animals scored for each condition. Source data are provided as a Source Data file.

the axonal protective function of TBC-10 may be specific for spectrin-induced injury.

The cytoskeletal spectrin-network forms tetramers composed of $\alpha$ - and $\beta$-spectrin aligned head-to-head ${ }^{18}$. The $n 493$ allele of unc-70/ $\beta$-spectrin contains an $\mathrm{L} 2044 \mathrm{P}$ substitution in its $17^{\text {th }}$ spectrin repeat, which is proposed to affect $\alpha \beta$-tetramerization ${ }^{6}$ and functions in a semi-dominant manner. Given the requirement of UNC-70/ $\beta$-spectrin dysfunction for axonal breakage to 
occur, we tested whether UNC-70/ $\beta$-spectrin was also acting in the surrounding epidermis to protect the axon from damage. We used a modified Mos 1 transposon (miniMos)-mediated strategy 19 to generate a transgenic strain carrying a single-copy of the semidominant unc-70(n493) allele tagged with mKate2 (unc-70(dn):: $m$ Kate2) and expressed selectively in either the epidermis or the touch receptor neurons, in the $t b c-10$ mutant background (Fig. 1h). Tissue-specific expression of UNC-70(dn)::mKate2 in the epidermis of $t b c-10$ mutant animals was sufficient to replicate the axonal breakage defect observed in the $t b c-10 ;$ unc-70 double mutants, whereas selective expression of the same dominant allele in the touch receptor neurons did not cause axonal breakage (Fig. 1h). Thus, UNC-70/ $\beta$-spectrin functions in the epidermis in synergy with TBC-10 to protect the PLM axon from damage.

To test if the UNC-70/ $\beta$-spectrin-induced defect in $t b c-10$ mutants was due to defects in tetramerization with $\alpha$-spectrin, we over-expressed a dominant-negative version of SPC-1/a-spectrin (SPC-1(dn)) containing the tetramerization domain ${ }^{6}$ in either the epidermis, or touch receptor neurons. Expression of $s p c-1(d n)$ in either tissue replicated buckling defects previously described in spectrin mutants (Supplementary Fig. $3 \mathrm{~B})^{6}$, indicating it may have disrupted spectrin tetramerization as hypothesized, however it was not sufficient to cause axonal breakage in a $t b c-10$ mutant background when expressed in either tissue (Supplementary Fig. 3A). Taken together these data suggest that together with TBC-10, UNC-70/ $\beta$-spectrin functions in the epidermis to protect the ensheathed PLM neurons from mechanical damage, and this function may be independent of $a$-spectrin.

TBC-10 and UNC-70/ $\beta$-spectrin localize to the membrane. To understand how TBC-10 protects the PLM axon from unc-70induced damage, we engineered an N-terminal $g f p:: 3 x$ Flag tag at the endogenous $t b c-10$ genomic locus using CRISPR-cas9 genome editing 20 . Using spinning disk confocal microscopy with deconvolution we visualized GFP::3xFlag::TBC-10 in large $z$-stacks, which were then rotated and viewed in an orthogonal plane to reveal the localization pattern of GFP::3xFlag::TBC-10 relative to the PLM neuron (labeled with a Pmec-17::tagRFP cell-specific marker; Fig. 2a-c). Importantly, the introduction of this tag did not cause axonal breakage in an unc-70(n493) background indicating that it is functional (Supplementary Table 1). In wild-type animals at the L4 stage, GFP::3xFlag::TBC-10 localized to the plasma membrane of the epidermis with a distinct and bright enrichment in a region directly apposed to the PLM axon, which corresponds to the epidermal furrow in which the PLM axon is ensheathed (Fig. 2a-c). An identical epidermal localization was observed in relation to the axon of the ALM neuron, which is also ensheathed within this tissue albeit in the dorsal, anterior region of the animal's body (Supplementary Fig. 4).

Given the sensitivity of $t b c-10$ mutants to UNC-70/ $\beta$-spectrin dysfunction, we visualized the localization of UNC-70(wt):: mKate 2 in the epidermis of wild-type animals. Using the above miniMos-mediated strategy, we generated a transgenic strain containing a single-copy insertion of a transgene expressing unc70(wt)::mKate2 under an epidermal-specific promoter and visualized its localization. This construct was able to partially rescue the breakage phenotype in tbc-10;unc-70 animals, suggesting that it is functional (Supplementary Fig. 3C). In wild-type animals, UNC-70(wt)::mKate2 localized to the epidermal membrane at the lateral midline where two epithelial tissues, the hypodermal hyp7 syncytium and the lateral seam cells, form adherens junctions (Fig. 2d). It also localized to a small number of puncta distributed throughout the epidermis between the apical and basal membranes (Fig. $2 \mathrm{~d}-\mathrm{f}$ ), as well as a large punctate structure adjacent to the PLM axon and the apical surface of the epidermis when viewed in an orthogonal section (Fig. $2 \mathrm{f}$ ), close to the site where hemidesmosomes form trans-epidermal attachments. Strikingly, overexpression of UNC-70(wt)::mKate2 in the $t b c-10$ mutant background was sufficient to induce axonal breakage, although at a reduced rate compared to the overexpression of UNC-70(dn)::mKate2 (Fig. 1h) or the $u n c-70(n 493)$ allele (Supplementary Fig. 3C). This phenotype was confirmed using a non-tagged version of wild-type UNC-70/ $\beta$-spectrin expressed tissue-specifically in the epidermis (Supplementary Fig. 3D). A null allele of unc-70/ $\beta$-spectrin in combination with $t b c-10$ mutants also caused an increase in the rate of axonal breakage (Supplementary Fig. 3E). In these animals, the increased rate of axonal breakage was less than in those carrying other alleles of $u n c-70$, an effect likely due to the reduced movement and very uncoordinated phenotype caused by the null allele. Indeed, consistent with this notion, RNAi-knockdown of unc-70 by feeding in $t b c-10$ mutants, which did not strongly affect movement, fully reproduced the axonal breakage defect (Supplementary Fig. 3F). This supports a model in which TBC-10 and $\mathrm{UNC}-70 / \beta$-spectrin are functioning at the epidermal membrane close to PLM to protect the ensheathed axon from damage. Furthermore, our data suggests that disruption to UNC-70/ $\beta$ spectrin in any form is sufficient to affect its function in this context.

TBC-10 regulates $\mathrm{RAB}-\mathrm{GTPase} \mathrm{RAB}-35$ activity to protect axons. $\mathrm{RAB}$ proteins are small GTPases that function as molecular switches to control a variety of intracellular trafficking pathways. Their activity is tightly controlled by the hydrolysis of GTP assisted by their specific GAPs and guanine exchange factors $(\mathrm{GEFs})^{21}$ (Fig. 3a). TBC-10 is a RAB-specific GAP which, in other contexts, functions with specificity for RAB-35 (Fig. 3a) ${ }^{22,23}$. We predicted that in the absence of functional TBC-10 there would be an increase in the active pool of RAB-35-GTP. If this was the case, and TBC-10 was functioning as a RAB-35-specific RabGAP, a loss-of-function mutation in $r a b$ - 35 would protect against degeneration in $t b c-10 ; u n c-70$ double mutants. Indeed, we found that a $r a b-35$ mutant strongly suppressed the axonal breakage phenotype of the $t b c-10 ; u n c-70$ double mutants (Fig. 3b). These results reveal that TBC-10 functions as a RAB-35-specific RabGAP and inactivates RAB-35 to protect touch receptor neurons from damage, in synergy with UNC-70/ $\beta$-spectrin.

Given that both TBC-10 and UNC-70/ $\beta$-spectrin are specifically required in the epidermis, we expected that RAB- 35 would also function in this tissue. To determine if this was the case, we tissue-specifically expressed a wild-type $r a b-35$ cDNA in the epidermis of $r a b-35 ; t b c-10 ; u n c-70$ triple mutants. As predicted, this transgene fully restored the axonal breakage phenotype (Fig. 3c), revealing that $\mathrm{RAB}-35$ also functions within the epidermis. To determine if this function of RAB-35 was conserved, we heterologously expressed a wild-type cDNA of human rab35 selectively in the epidermis. Remarkably, the human ortholog also fully restored the axonal breakage phenotype, suggesting that the function of RAB-35 is conserved across species (Fig. $3 \mathrm{~d}$ ).

To demonstrate that TBC-10 normally functions to increase the GTPase activity of RAB-35, we tested the effect of expressing predicted GTP- and GDP-locked versions of RAB-35 on axonal breakage. Tissue-specific overexpression of RAB-35(Q69L), predicted to lock RAB-35 in the GTP-bound conformation, in the epidermis of $r a b-35 ; t b c-10 ; u n c-70$ triple mutants restored the axonal breakage phenotype (Fig. 3c). Furthermore, overexpression of a RAB-35(S24N) GDP-locked form tissue-specifically in the epidermis of $t b c-10 ; u n c-70$ mutant animals was able to rescue the axonal breaks (Fig. 3c). Remarkably, we oberved identical 



Fig. 2 TBC-10 and UNC-70/ $\beta$-spectrin localize to the epidermal

membrane. a Deconvolved spinning disk confocal maximum projection and scheme of GFP::3xFlag::TBC-10 localization in a typical wild-type animal with the PLM neuron labeled with a cytosolic TagRFP (Pmec-17::tagRFP; magenta). Arrows indicate regions of the epidermal furrow visible in a lateral perspective. $\mathbf{b}$ An orthogonal perspective and scheme of GFP::3xFlag::TBC-10 localization in the projected $z$-stack shown in a. c A high-magnification image and scheme of the boxed area in $\mathbf{b}$. These micrographs are representative of the GFP::3xFlag::TBC-10 localization seen in $N=12$ animals at the L4 stage. $\mathbf{d}$ Image and scheme of UNC-70::mKate2 localization (magenta) expressed under an epidermal-specific promoter in a typical wild-type animal with the PLM neuron labeled with a cytosolic GFP (Pmec-4::GFP). e An orthogonal perspective and scheme of UNC-70:: mKate2 localization in the projected $z$-stack shown in $\mathbf{d}$. $\mathbf{f} A$ highmagnification image and scheme of the boxed area in $\mathbf{e}$. These micrographs are representative of the UNC-70::mKate2 localization seen in $N=6$ animals at the L4 stage. Scale bars are $25 \mu \mathrm{m}$ in $\mathbf{a}, 5 \mu \mathrm{m}$ in $\mathbf{b}$, and $2 \mu \mathrm{m}$ in c.

effects of GTP- and GDP-locked versions of human RAB35 when expressed heterologously in the epidermis (Fig. 3d). These data are consistent with TBC-10 functioning as a RAB-35-specific GAP in this context. In vivo, conversion of RAB-35-GDP to RAB-35-GTP is catalyzed by specialized GEFs. In C. elegans RME-4 and FLCN-1, orthologs of mammalian connecden and folliculin, respectively ${ }^{14}$, have been demonstrated to function as GEFs for RAB-35 (Fig. 3a) ${ }^{22-24}$. In the tbc-10;unc-70 background, loss of function in rme-4 partially rescued the axonal breakage phenotype, whereas flcn-1 loss-of-function had no effect (Fig. 3e). Axonal breakage in flcn-1;rme-4 double mutants in the tbc-10; unc-70 background was indistinguishable from rme-4 mutants, suggesting RME-4 functions as the major GEF for RAB-35 in this context (Fig. 3e). Given the partial rescue of rme-4 loss-offunction, we cannot exclude the role in this context of another unknown GEF, in addition to RME-4. These data confirm TBC10 and RME-4 function to regulate RAB-35 activity within the epidermis, which when overactive leads to axonal breakage in an unc-70 mutant background.

We next asked where RAB-35 localized within the epidermis to modulate the axonal damage seen in tbc-10;unc-70 double mutants. To investigate this we expressed and visualized RAB35::mKate2 selectively in the epidermis of rab-35(b1013) mutant animals (Fig. 3f-h). RAB-35::mKate2 was enriched in punctate, tubular and spherical structures close to the apical membrane of the epidermis and, similar to TBC-10, at the epidermal furrow (Fig. 3f-h). Therefore, RAB-35 is normally localized close to the PLM axon in the epidermal membrane, is enriched in the furrow surrounding the PLM axon, and TBC-10 loss-of-function sensitizes the axon to spontaneous breakage by increasing the active pool of RAB-35-GTP.

UNC-70/ $\beta$-spectrin and TBC-10 regulate hemidesmosomes. The pattern of TBC-10, UNC-70 and RAB-35 localization in regions of the epidermis overlying the muscle was reminiscent of fibrous structures, called hemidesmosomes, which mechanically couple the muscle to the cuticle via the epidermis (Fig. 2a, d and Fig. $3 f^{25}$. Similar structures also transmit mechanical deformation of the cuticle to the touch receptor neurons ${ }^{25-29}$. This led us to hypothesize that the attachment between the touch receptor neuron PLM and its surrounding epidermis via hemidesmosomes may be disrupted in $t b c-10 ; u n c-70$ mutants and drive the axonal breakage phenotype. To test this possibility, we visualized the localization of VAB-10a/spectraplakin, a core component of $C$. elegans hemidesmosomes and the sole ortholog of vertebrate plectin and BPAGle ${ }^{29}$. In wild-type animals at the L4 stage, a functional CRISPR-Cas9 edited VAB-10a::GFP localized to epidermis-muscle and axon-epidermis attachment sites in a circumferentially striped and periodic pattern (Fig. 4a and Supplementary Fig. 5A-D) as previously reported ${ }^{29,30}$. Twenty-four hours later, in 1-day-old adult (1DOA) animals, the periodicity of VAB-10a::GFP localization was lost and VAB-10a::GFP was continuously distributed along the length of the PLM axon in wild-type animals (Fig. $4 \mathrm{~b}$ and Supplementary Fig. 5A-D). This change in localization pattern may reflect a remodeling event within hemidesmosomes at this stage. As predicted, tbc-10;unc-70 mutants presented with defects in the attachment of the PLM axon to the surrounding epidermis. This was evident in the L4 stage before the appearance of axonal breakage, where there was an increase in the proportion of animals missing VAB-10a:: GFP-positive puncta along the length of the axon in localized regions compared to wild-type animals (Fig. $4 \mathrm{c}$, e). The defect worsened in 1DOA animals where the localization of VAB-10a:: GFP became discontinuous and large gaps appeared in regions of intact and broken PLM axons (Fig. $4 \mathrm{~d}, \mathrm{f}$ ). In the example shown in Fig. 4d, we observed a region of almost complete loss of VAB10a::GFP combined with buckles in the PLM axon. Importantly, we did not observe buckles or breaks in regions with continous attachment, nor did we observe any changes to the periodicity or spacing of VAB-10a::GFP-positive puncta in regions where attachment was present (Supplementary Fig. 5B, C). Single mutants for $t b c-10$ and $u n c-70$ had normal attachment (Supplementary Fig. 6A-F), although the levels of VAB-10a::GFP were reduced in unc-70 single mutants compared to wild-type animals in 1DOA (Supplementary Fig. 5B). These data demonstrate that hemidesmosome attachment structures are disrupted in $t b c-10$; 
a
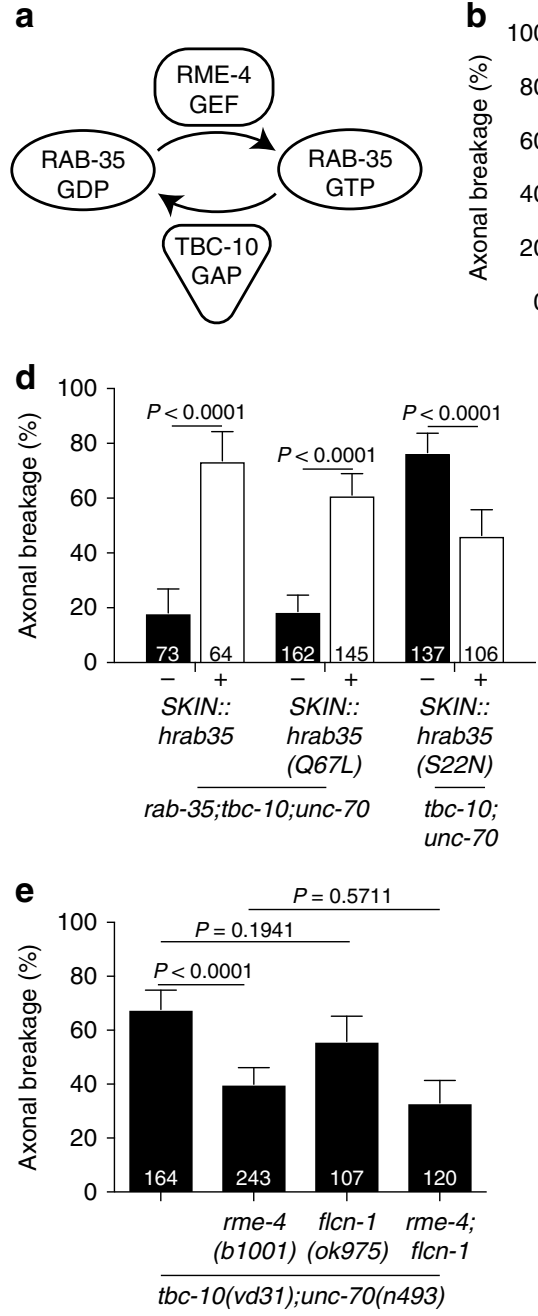
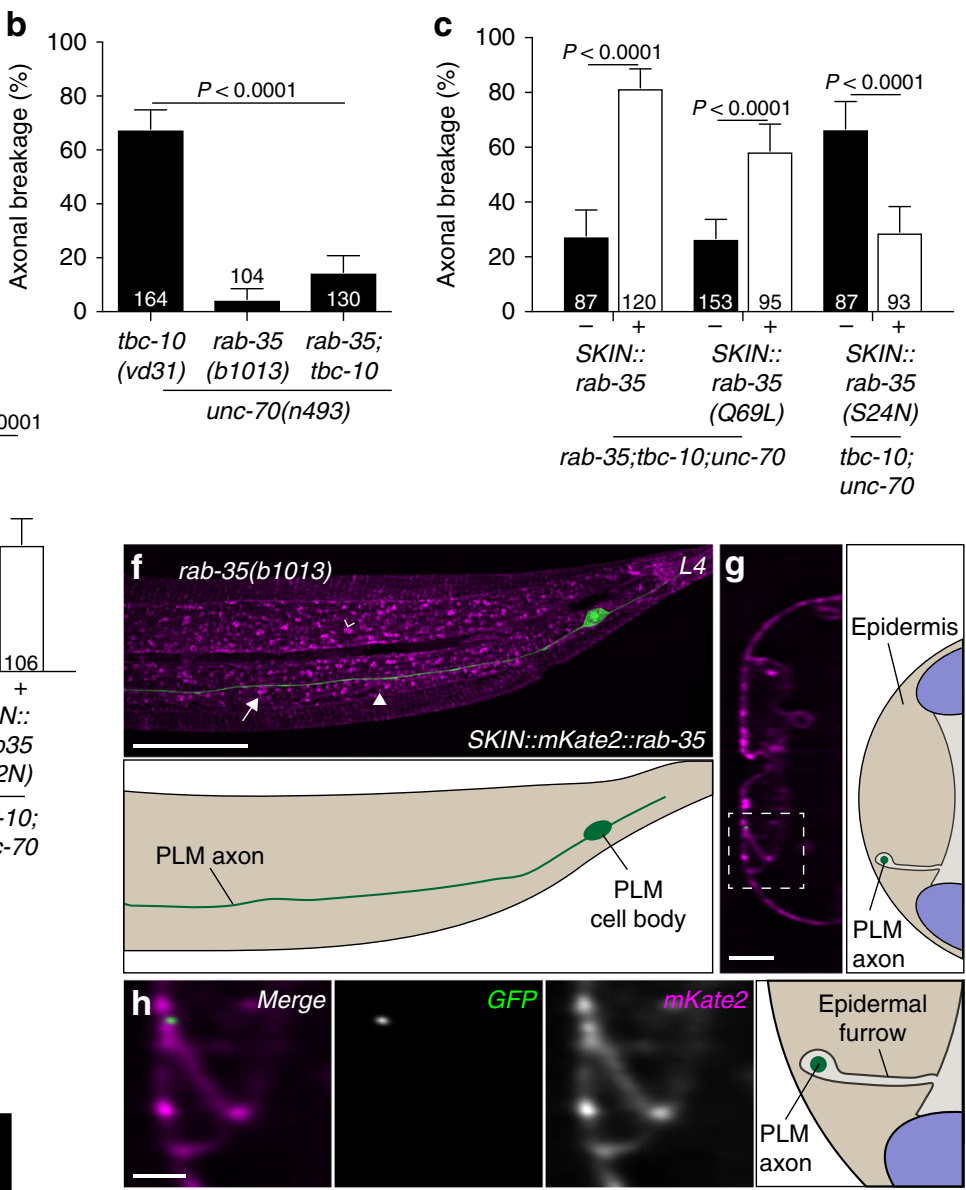

Fig. 3 TBC-10 is a RAB-35-specific GAP. a Scheme of the activation cycle of RAB-35. b Mean penetrance of axonal breaks in unc-70(n493) mutants with and without mutations in $t b c-10(v d 31)$ and rab-35(b1013). c Mean penetrance of axonal breakage in rab-35(b1013); tbc-10(vd31); unc-70(n493) or tbc-10(vd31); unc-70(n493) mutants carrying transgenes expressing either the wild-type $C$. elegans rab-35, a constitutively active rab-35(Q69L) mutant, or a constitutively inactive rab-35(S24N) mutant under the control of an epidermal-specific promoter, versus respective non-transgenic siblings. $\mathbf{d}$ Mean penetrance of axonal breakage in rab-35(b1013); tbc-10(vd31);unc-70(n493) or tbc-10(vd31);unc-70(n493) mutants carrying transgenes expressing either the wild-type human rab35, a constitutively active human rab35(Q67L) mutant, or a constitutively inactive human rab35(S22N) mutant under the control of an epidermal-specific promoter, versus their respective non-transgenic siblings. e Mean penetrance of axonal breaks in tbc-10(vd31); unc-70(n493) mutants, with and without single or double mutants for rme-4 and flcn-1. P-values in $\mathbf{b}$ and $\mathbf{e}$ are determined from a one-way ANOVA with a Tukey multiple-comparison of proportions using an Agresti-Coull interval. P-values in $\mathbf{c}$ and $\mathbf{d}$ determined from a comparison of proportions using an Agresti-Coull interval. Error bars indicate a $95 \%$ confidence interval. Source data are provided as a Source Data file. $\mathbf{f}$ Deconvolved spinning disk confocal maximum projection and scheme of mKate2:: RAB-35 localization (magenta) expressed under an epidermal-specific promoter in a typical rab-35 mutant animal with the PLM neuron labeled with a cytosolic GFP (Pmec-4::GFP). Tubular, punctate, and spherical structures labeled with an arrow, closed arrowhead, and open arrowhead respectively. $\mathbf{g}$ An orthogonal perspective and scheme of mKate2::RAB-35 localization in the projected $z$-stack shown in $\mathbf{f}$. $\mathbf{h}$ A high-magnification image and scheme of the boxed area in $\mathbf{g}$. These micrographs are representative of the mKate2::RAB-35 localization seen in $N=5$ animals at the L 4 stage. Scale bars are $25 \mu \mathrm{m}$ in f, $5 \mu \mathrm{m}$ in $\mathbf{g}$, and $2 \mu \mathrm{m}$ in $\mathbf{h} . \mathrm{N}$-values are indicated on bar graphs and represent the number of individual animals scored for each condition.

unc-70 axons prior to axon breakage and partially lost in adult animals.

In addition to the VAB-10a::GFP localization, we investigated the localization of LET-805/myotactin, a key adhesion molecule containing multiple fibronectin repeats predicted to link VAB$10 \mathrm{a}$ to integrins on the muscle and the PLM axon on the basal side of the epidermis ${ }^{27,31}$. Consistent with our VAB-10a findings, a CRISPR-Cas9 edited let-805:::wrmScarlet localized to periodic puncta along the PLM axon in L4 stage wild-type animals (Fig. 5a and Supplementary Fig. 5E-H). The tagged LET-805::wrmScarlet was deemed functional as animals were viable. In $1 \mathrm{DOA}$ wildtype animals the periodic localization was lost, with a more uniform and continuous distribution observed along the axon (Fig. 5b and Supplementary Fig. 5E-H). As with VAB-10a, LET805::wrmScarlet localization revealed a loss of attachment of the PLM axon to the surrounding epidermis in $t b c-10$; unc-70 animals. At the L4 stage there was an increase in the proportion of animals missing LET-805::wrmScarlet-positive puncta along the length of the axon in localized regions compared to wild-type animals (Fig. 5c, e). In 1DOA tbc-10;unc-70 animals we observed large gaps in the localization of LET-805::wrmScarlet in intact and broken PLM axons (Fig. 5d, f). In tbc-10 and unc-70 single mutants, LET-805::wrmScarlet was localized along the PLM axon in an identical pattern to wild-type animals (Supplementary 

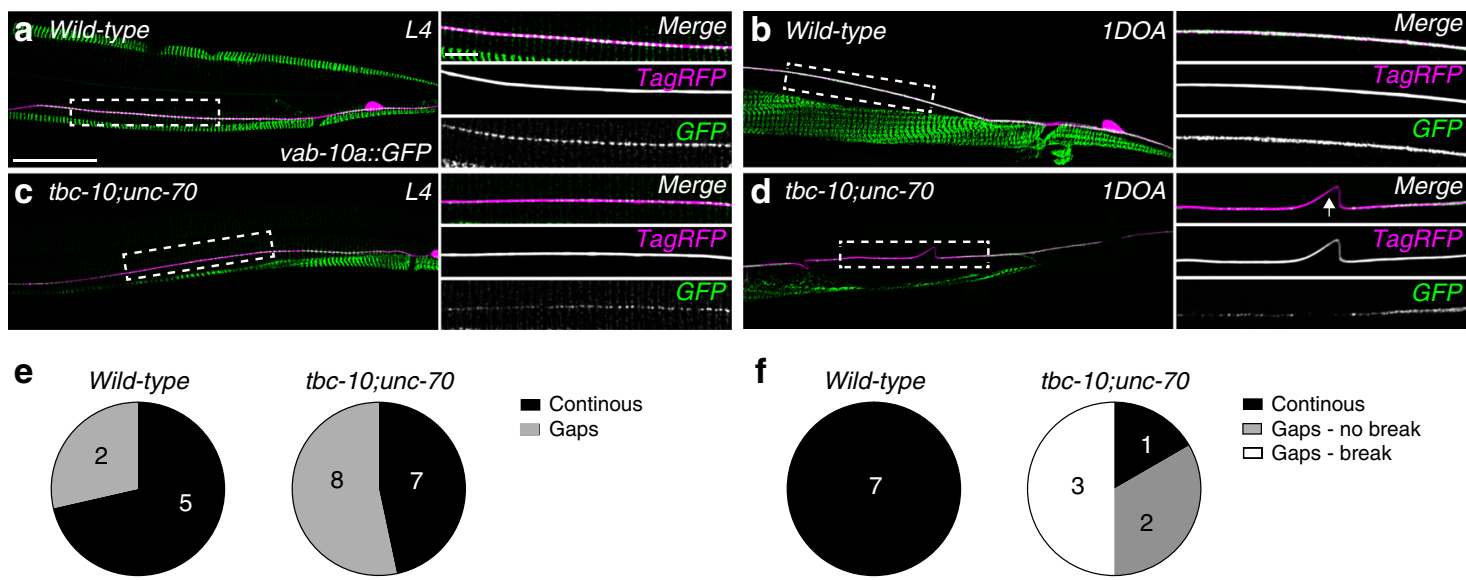

- Continous

$\square$ Gaps - no break

$\square$ Gaps - break

Fig. 4 VAB-10a/spectraplakin localization is disrupted in tbc-10;unc-70 mutants. a, b Deconvolved spinning disk confocal maximum projection of typical endogenous VAB-10a::GFP localization at the L4 (a) and 1-day-old adult (1DOA) (b) stage in wild-type animals from a lateral perspective, with the PLM neuron labeled with a cytosolic TagRFP (Pmec-17::tagRFP, magenta). The side panels in a-d show the boxed area indicated in the left panel as a merged image and individual channels. c, d Deconvolved spinning disk confocal maximum projection of typical endogenous VAB-10a::GFP localization at the L4 (c) and 1DOA (d) stage in tbc-10(vd31); unc-70(n493) animals from a lateral perspective, with the PLM neuron labeled with a cytosolic TagRFP (Pmec-17:: tagRFP, magenta). Arrow in $\mathbf{d}$ indicates a buckle in the PLM axon. Scale bars in a are $25 \mu \mathrm{m}$ and $5 \mu \mathrm{m}$ in the left and side panels, respectively. e Normalized intensities of VAB-10a::GFP along a $50 \mu \mathrm{m}$ of the PLM axon at the L4 stage were scored as containing gaps (defined as $>5$ puncta with intensity values < 0.2 a.u.) or continuous attachment (defined as $<5$ puncta with intensity values $<0.2$ a.u.). $\mathbf{f}$ VAB-10a::GFP localization at the 1 DOA stage was scored as continuous (no gaps in localization greater than $10 \mu \mathrm{m}$ ) or containing gaps (a region of no localization greater than $10 \mu \mathrm{m}$ ) with and without axonal breakage. $\mathrm{N}$-values are indicated on charts and represent the number of individual animals scored for each condition.
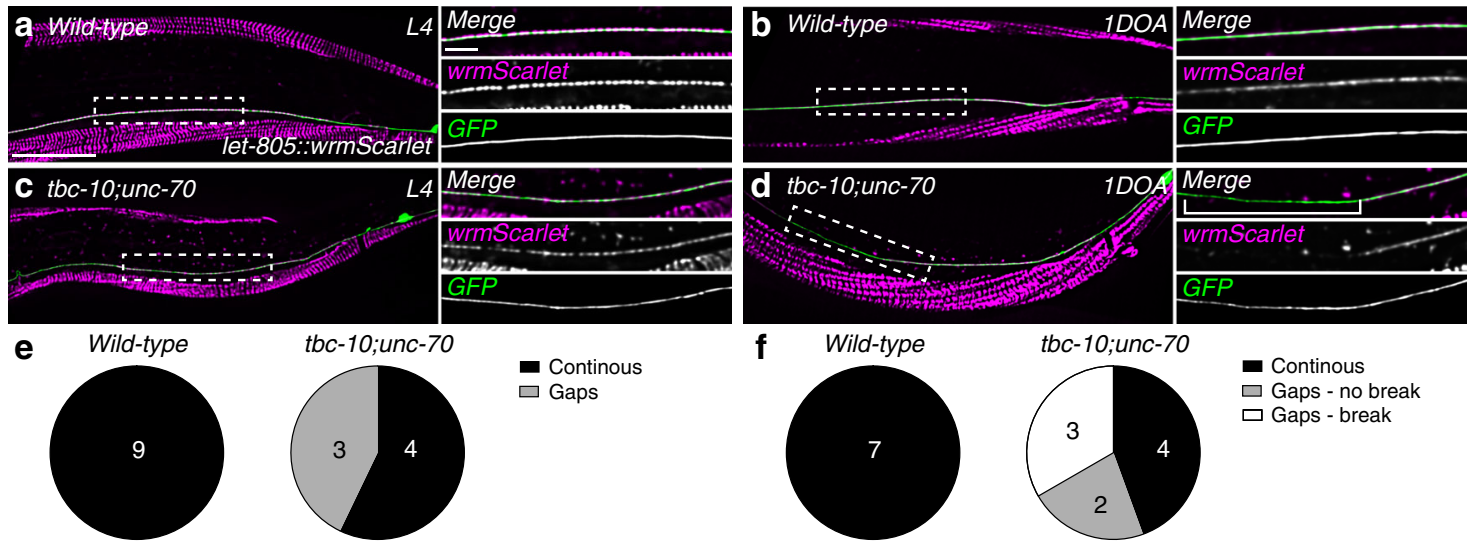

Fig. 5 LET-805/myotactin localization is disrupted in tbc-10;unc-70 mutants. a, b Deconvolved spinning disk confocal maximum projection of typical endogenous LET-805::wrmScarlet localization (magenta) at the L4 (a) and 1-day-old adult (1DOA) (b) stage in wild-type animals from a lateral perspective, with the PLM neuron labeled with a GFP (Pmec-4::GFP). The side panels in a-d show the boxed area indicated in the left panel as a merged image and individual channels. c, d Deconvolved spinning disk confocal maximum projection of typical endogenous LET-805::wrmScarlet localization (magenta) at the L4 (c) and 1DOA (d) stage in tbc-10; unc-70 animals from a lateral perspective, with the PLM neuron labeled with a cytosolic GFP (Pmec4::GFP). Open bracket in side panel $\mathbf{d}$ indicates the region where LET-805::wrmScarlet localization is absent. Scale bars in $\mathbf{a}$ are $25 \mu \mathrm{m}$ and $5 \mu \mathrm{m}$ in the left and side panels, respectively. e Normalized intensities of LET-805::wrmScarlet along a $50 \mu \mathrm{m}$ of the PLM axon at the L4 stage were scored as containing gaps (defined as $>5$ puncta with intensity values $<0.2$ a.u.) or continuous attachment (defined as $<5$ puncta with intensity values $<0.2$ a.u.). $\mathbf{f}$ LET- 805 : wrmScarlet localization at the 1DOA stage was scored as continuous (no gaps in localization greater than $10 \mu \mathrm{m}$ ) or containing gaps (a region of no localization greater than $10 \mu \mathrm{m}$ ) with and without axonal breakage. $\mathrm{N}$-values are indicated on charts and represent the number of individual animals scored for each condition.

Fig. 6G-L). Interestingly, each single mutant had opposing effects on the membrane levels of LET-805::wrmScarlet in each stage. At the L4 stage $u n c-70$ animals had a reduced intensity of LET-805:: wrmScarlet (Supplementary Fig. 5F), while in 1DOAs tbc-10 mutants displayed an increase in the level of LET-805::wrmScarlet at the membrane compared to wild-type animals (Supplementary Fig. 5F).

Combined with the strong genetic data presented, these results indicate that $t b c-10$ and $u n c-70$ mediate parallel pathways in maintaining attachment of the PLM axon to the surrounding epidermal tissue, which is critical for axonal integrity. Importantly, the disruption observed in the localization of both VAB10a::GFP and LET-805::wrmScarlet in tbc-10;unc-70 doublemutant animals at the L4 stage preceded, and was predictive of, future breakage events (Supplementary Fig. 6M-P). Therefore our results support a model in which the PLM axon breaks as a consequence of the partial loss of hemidesmosome mediated attachment between the axon and its surrounding epidermis. 
Strain of movement induces loss of hemidesmosomes in mutants. C. elegans hemidesmosomes have been suggested to function as mechanosensors during epithelial morphogenesis and respond to tension by activating signaling pathways ${ }^{32}$. To confirm that the axonal breaks were induced by the mechanical strain of movement, we took advantage of unc-54(e1009) mutant animals, which carry a mutation in a muscle myosin class II heavy chain and as a consequence are severely paralyzed. In the absence of movement, the axons of unc-54;tbc-10;vdSi2(Pdpy-7::unc-70 $(d n):: m K a t e 2)$ animals did not break, suggesting that the axonal breakage phenotype is a consequence of movement (Fig. 6a). Consistent with this model, a gain-of-function mutation in the adenylate cyclase $a c y-1$ that induces a hyperactive phenotype ${ }^{33}$ was able to increase the rate of axonal breakages in $t b c-10 ; v d S i 2$ (Pdpy-7::unc-70(dn)::mKate2) animals (Fig. 6a). Conversely, a gain-of-function mutation in the cGMP-dependent protein kinase gene egl-4, which causes increased periods of quiescence ${ }^{34}$, reduced the rate of axonal breakage in $t b c-10$; vdSi2(Pdpy-7::unc$70(d n):: m K a t e 2)$ animals (Fig. 6a). In principle, defects in hemidesmosomes could arise independently of movement as a consequence of UNC-70/ $\beta$-spectrin and TBC-10 dysfunction, and movement provides only the mechanical force required to induce damage in detached axons. Alternatively, UNC-70/ $\beta$-spectrin and TBC-10 may function to ensure hemidesmosomes are mechanically resistant. In this case loss of hemidesmomes would only occur following movement. To distinguish between these possibilities we visualized VAB-10a::GFP localization in $u n c-54 ; t b c-10$; $v d S i 2(P d p y-7:: u n c-70(d n):: m K a t e 2)$ paralyzed animals. Intriguingly, VAB-10a::GFP attachment sites remained intact and continuous in paralyzed animals at the $1 \mathrm{DOA}$ stage (Fig. $6 \mathrm{~b}, \mathrm{c}$ ). These data are consistent with UNC-70/ $\beta$-spectrin and TBC-10 functioning within the epidermis to maintain mechanically resistant hemidesmosomes and axon-epidermis attachment during movement (Fig. 7).

\section{Discussion}

To maintain a functional nervous system, circuits must be able to withstand varying degrees of mechanical strain during their lifetime. Using a forward genetic approach we have identified a key protection mechanism for a $C$. elegans sensory axon that allows their attachment to be maintained during mechanical strain and protects from strain-induced degeneration. We have several lines of evidence to support this model. Firstly, we show that $t b c-10 ; u n c-70$ mutants undergo spontaneous axonal degeneration in response to normal body movement. Secondly, we demonstrate that UNC-70/ $\beta$-spectrin and the Rab-GTPaseactivating protein TBC-10 function non-cell autonomously and synergistically in the epidermis to maintain hemidesmosomes at sites of axon-epidermal attachment. Thirdly, loss of hemidesmosomes in $t b c-10 ; u n c-70$ mutants is induced by movementindicating that their normal function is to maintain these fibrous organelles under conditions of increased strain.

The cytoskeletal spectrin-network is thought to mediate strain resistance intrinsically within axons by maintaining tension via the actin-spectrin cytoskeleton 6,7 . In the nematode C. elegans, disruption of the cytoskeletal spectrin-network causes axons to buckle and deform during body movement, leading to spontaneous axonal breakage ${ }^{6,9,10}$. Until recently, most data supported a model in which the spectrin-network functioned largely cellautonomously within neurons to protect them from damage. However, it is becoming increasingly clear that $\beta$-spectrin has a much larger role in neuronal support cells than was previously appreciated. Here, we uncover a role for $\beta$-spectrin in the maintenance of axonal integrity, whereby it functions non-cellautonomously in the epidermis surrounding a mechanosensory axon to regulate adhesion. We have some evidence to suggest this role is potentially independent of its teteramerization partner, $a$ spectrin, as the predicted disruption to the spectrin-network with a dominant-negative $\alpha$-spectrin was not sufficient to cause axonal damage. There are multiple examples of $\beta$-spectrin interacting with integral membrane proteins and cell adhesion molecules ${ }^{35}$. $\beta$-spectrin directly interacts with the neural cell adhesion molecule $\mathrm{NCAM}^{36,37}$, as well as with CHL1, a close homolog of the immunoglobulin superfamily cell adhesion molecule $\mathrm{L1}^{38}$, and is required independently of $a$-spectrin for the polarized localization of the $\mathrm{Na}^{+} / \mathrm{K}^{+}$ATPase in the epithelia of the Drosophila midgut $^{39}$. On its own, disruption to unc-70/ $\beta$-spectrin causes only a mild axonal breakage phenotype; however, in combination with mutations in the Rab-GAP $t b c-10$, and subsequent increases in RAB-35 activity, it causes loss of hemidesmosomes, detachment of the axon from the surrounding epidermis, and axonal degeneration. The PLM touch receptor neurons of $C$. elegans are unmyelinated and lack specialized glia. Instead, they have an intimate and complex relationship with the overlying epidermal tissue, which is essential for the correct development of the PLM neuron ${ }^{11}$ and, as we demonstrate here, the maintenance of its integrity. In this respect, the relationship resembles that of specialized myelinating glia, oligodendrocytes and Schwann cells, which wrap their membrane around axons to provide insulation and trophic support, sculpt structure, and cluster ion channels ${ }^{40}$. In C. elegans, the spectrin-network has been demonstrated to partially function non-cell-autonomously within the epidermis to limit buckling of touch receptor neuron axons during normal body movement, where it was postulated to affect membrane elasticity ${ }^{6,7}$. Moreover, in vertebrate myelinating glia the spectrinbased cytoskeleton has been implicated in the formation and maintenance of adhesion complexes at the paranodal regions flanking nodes of Ranvier ${ }^{41,42}$, and disruption of axon-glia attachments causes axonal degeneration in Purkinje neurons ${ }^{43}$. In this context, together with the data we present here, this suggests mechanisms governing adhesion play highly conserved roles in axonal protection.

Our results illuminate a complex and intricate system regulating tissue attachment and neuronal maintenance in response to movement-induced mechanical strain. In mutants where attachment pathways are completely disrupted, the PLM axon fails to become ensheathed within the epidermis and instead resides between the muscle and epidermis where it originally develops ${ }^{11}$. We have previously shown that preventing attachment and ensheathing of the PLM axon within the epidermis is not sufficient to cause axonal degeneration ${ }^{44}$. A potential explanation for these seemingly contradictory phenotypes is that in the case of uniform attachment (or uniform detachment) of the axon and epidermis, the axon is able to withstand compression from body movement due to intrinsic protection mechanisms. However, we propose that partial detachment may be detrimental because of an imbalance in tension and force experienced by the axon. Here, we demonstrate that following normal development, hemidesmosomes are susceptible to subtle changes, which render them, and consequently the ensheathed axon, vulnerable to mechanical stress and degeneration. The axonal process of PLM is coupled to the surrounding epithelia via mechanically resilient adhesion complexes, which act like shock absorbers to both transduce force from the cuticle to the axon for touch sensation ${ }^{26}$, as well as minimizing the damaging compressive forces experienced by the axon during body movement. Hemidesmosomes have been postulated to facilitate tissue sliding while maintaining normal attachment by dynamic assembly and disassembly during tissue movement ${ }^{45}$, and are highly susceptible to subtle perturbations in the levels of their components and the dynamics of their assembly and disassembly ${ }^{46}$. Hemidesmosomes have also 

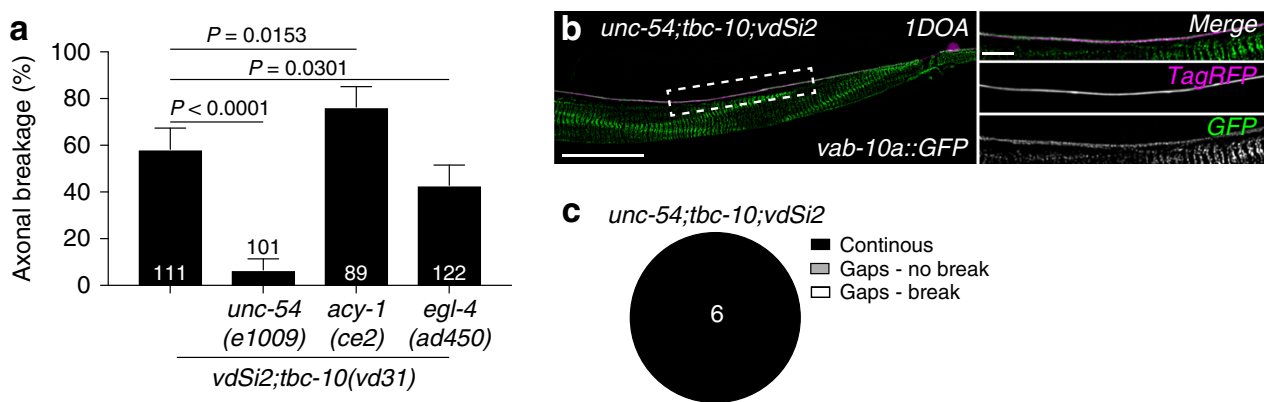

C

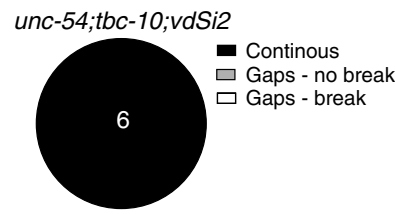

Fig. 6 Attachment defects and axonal breakage are movement dependent. a Mean penetrance of axonal breaks in tbc-10(vd31);vdSi2(Pdpy-7::mKate2::unc70(n493)) animals with and without mutations in unc-54, acy-1, and egl-4. b Deconvolved spinning disk confocal maximum projection of typical endogenous VAB-10a::GFP localization in 1-day-old adult (1DOA) unc-54;tbc-10; vdSi2 animals from a lateral perspective, with the PLM neuron labeled with a cytosolic TagRFP (Pmec-17::tagRFP; magenta). The side panels in $\mathbf{b}$ show the boxed area indicated in the left panel as a merged image and individual channels. Scale bars in $\mathbf{b}$ are $25 \mu \mathrm{m}$ and $5 \mu \mathrm{m}$ in the left and side panels, respectively. c VAB-10a::GFP localization at the 1DOA stage was scored as continuous (no gaps in localization greater than $10 \mu \mathrm{m}$ ) or containing gaps (a region of no localization greater than $10 \mu \mathrm{m}$ ) with and without axonal breakage. $P$-values in a are determined from a one-way ANOVA with a Tukey multiple-comparison of proportions using an Agresti-Coull interval. Error bars indicate a 95\% confidence interval. $\mathrm{N}$-values are indicated on bar and pie charts and represent the number of individual animals scored for each condition. Source data are provided as a Source Data file.

a

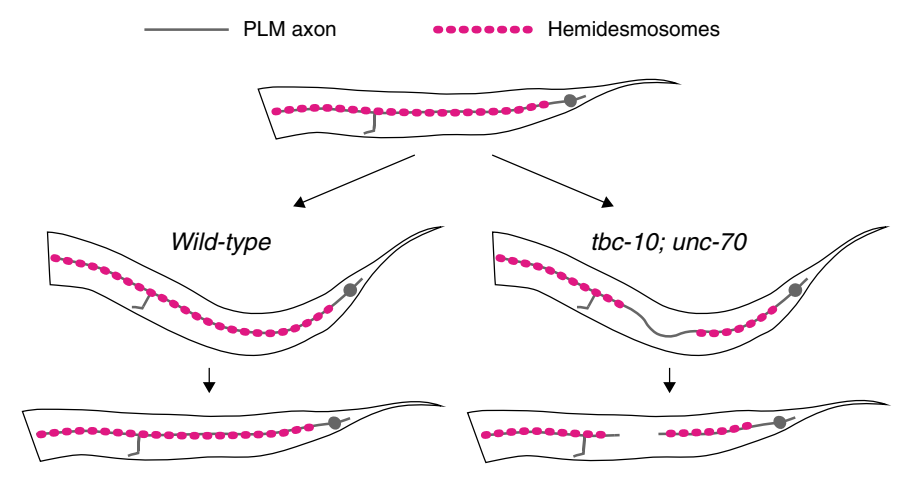

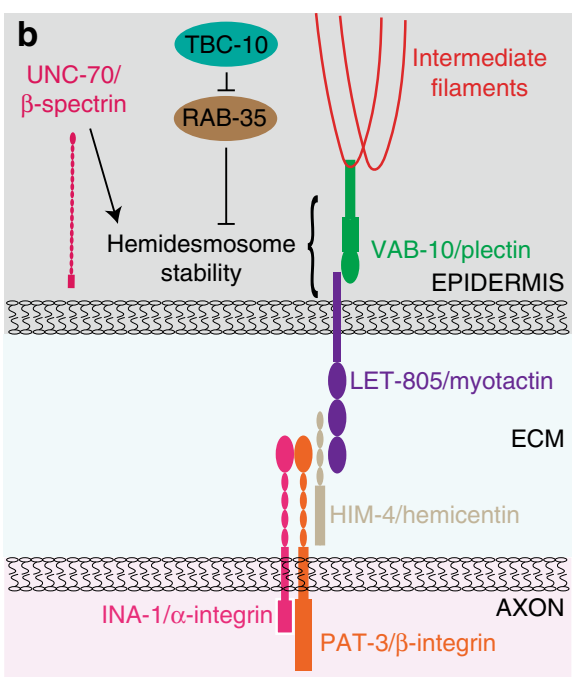

Fig. 7 Proposed model for attachment defects leading to axonal degeneration. a During normal body movement hemidesmosomes are mechanically resistant and attachment is maintained along the length of the axon in wild-type animals. In tbc-10;unc-70 mutants, these attachments are no longer mechanically resistant and the strain of movement leads to the intermittent loss of hemidesmosomes. Successive rounds of movement leads to increased local strain in the now detached axon, causing axonal breakage and degeneration. $\mathbf{b}$ Schematic representation of known and proposed components of hemidesmosomes linking the axons of touch receptor neurons to the epidermis. We propose that UNC-70/ $\beta$-spectrin, the Rab-GTPase-activating protein TBC-10 and its RAB-35 target, maintain axonal integrity by functioning non-cell autonomously in the epidermis to control the stability of hemidesmosomes in response to mechanical strain via parallel pathways.

been shown to act as mechanosensors during epithelial morphogenesis, relaying muscle tension to intiate intracellular signaling processes ${ }^{32}$ and are remodeled in response to stretch ${ }^{47}$. Importantly, loss of hemidesmomes and subsequent axonal breakage in $t b c-10$; unc-70 mutants was prevented by paralysis. We propose that the mechanical strain of body movement is transmitted via hemidesmosomes into the epidermis, where TBC10 and UNC-70/ $\beta$-spectrin function redundantly to maintain mechanically resistant hemidesmosomes, potentially by participating in remodeling or stabilization of these structures to prevent their disassembly (Fig. 7b). Indeed, TBC-10 and RAB-35 have known roles in membrane recycling events ${ }^{14,24}$ and $\beta$ spectrin has been demonstrated to both stabilize membrane proteins in epithelia ${ }^{39}$ and adhesion complexes mediating axonglia attachment ${ }^{41-43}$. Consistent with this notion, our results indicate that UNC-70 and TBC-10 can regulate the levels and balance of the hemidesmosome components on the membrane, with mutations in $u n c-70$ causing a decrease of both VAB-10 and LET-805, whereas mutations in $t b c-10$ cause an increased level of LET-805. Our results paint a picture of axonal adhesion as a finely balanced and tightly regulated protective mechanism that contributes to the mechanical resilience of axonal compartments.

\section{Methods}

Strains. All nematodes were cultured on nematode growth medium (NGM) plates seeded with Escherichia coli OP50 according to standard methods ${ }^{48}$. Any strain containing the unc-70(n493) or unc-70(s1502) alleles were cultured in the same conditions with Escherichia coli HB101. Unless otherwise noted, experiments were performed on 1-day-old adults raised at $22^{\circ} \mathrm{C}$, selected $24 \mathrm{~h}$ prior at the $\mathrm{L} 4$ stage. The wild-type N2 Bristol strain was used with the alleles and transgenes listed in Supplementary Tables 1 and 2. The morphology marker uIs115(Pmec-17::tagRFP)] 
was a gift from Martin Chalfie, unc-70(s1502) and oxIs95(Ppdi-2::unc-70) were gifts from Erik Jorgensen, and pgIs10[Pmec-17::spc-1(1-170)::mCherry] was a gift from Miriam Goodman.

Isolation of genetic mapping of mutant. Animals of genotype unc-70(s1502); oxIs95(Ppdi-2::unc-70);zdIs5 were mutagenized using $50 \mathrm{mM}$ ethyl methanesulfonate (EMS; Sigma) for $4 \mathrm{~h}$. The $v d 31$ mutation was isolated from a non-clonal F2 progeny screen. The $v d 31$ allele was crossed into an unc-70(n493);zdIs5 background and backcrossed $\mathrm{x} 3$ with N2 Bristol worms before whole-genome sequencing was conducted (Queensland Brain Institute, Center for Brain Genomics) on QH5436 (vd31;unc-70(n493);zdIs5), QH3631(unc-70(s1502);oxIs95;zdIs5), OH5343(unc-70 (n493);zdIs5) and the N2 Bristol strain at a mean depth of $\geq 58$. Mapping and calling of variants was performed using CloudMap 49,50 by comparing the frequency of likely EMS mutations across the genome ${ }^{51}$ after background subtraction.

\section{Molecular biology, CRIPSR-Cas9 gene editing, and single-copy insertions} Standard molecular biology techniques were used ${ }^{52}$. All plasmids were generated using either classic restriction enzyme digestion followed by ligation or Gibson assembly $y^{53,54}$ and details of their construction are listed in Supplementary Table 3.

Gene-edited $t b c$ - 10 knock-in strains were generated using CRISPR-Cas9 gene editing with a self-excising cassette ${ }^{20}$. Approximately $650 \mathrm{bp}$ homology arms were inserted into the GFP^SEC^3xFlag vector pDD282 (a gift from Bob Goldstein; Addgene plasmid \#66823) using Gibson assembly (amplified from N2 genomic DNA). A Cas9 target site was chosen using the MIT CRISPR design tool (http:// crispr.mit.edu) and inserted into a Cas9-sgRNA vector pDD162 (a gift from Bob Goldstein; Addgene plasmid \#47549) using QuikChange Site-directed Mutagenesis (Agilent Technologies, USA). To generate lines, $10 \mathrm{ng} / \mu \mathrm{L}$ of the homologous repair template and $50 \mathrm{ng} / \mu \mathrm{L}$ of the Cas9-sgRNA construct containing targeting sequence, as well as $10 \mathrm{ng} / \mu \mathrm{L}$ pGH8, $5 \mathrm{ng} / \mu \mathrm{L}$ pCFJ104, and $2.5 \mathrm{ng} / \mu \mathrm{L}$ pCFJ90 (gifts from Erik Jorgensen; Addgene \#19359, \#19328 and \#19327) were injected into the gonads of young adult animals. Injected animals were transferred to new OP50 plates (three per plate) and allowed to generate progeny for 3 days at $25^{\circ} \mathrm{C}$. Then, hygromycin was added to a final concentration of $250 \mu \mathrm{g} / \mathrm{mL}$ and placed at $25^{\circ} \mathrm{C}$ for another 4 days. Animals that survived hygromycin selection, were rollers and lacked fluorescent injection markers were singled onto fresh OP50 plates to establish lines. A knock-in let-805(syb380) allele with a C-terminal wrmScarlet tag was obtained from Suny Biotech (China).

miniMos NeoR vectors were constructed using Gibson assembly of two PCR fragments to insert the construct of interest into the miniMos NeoR (pCFJ910 was a gift from Erik Jorgensen; Addgene plasmid \#44481). Single-copy insertions ${ }^{19}$ were generated by injecting young adult animals with miniMos-based vectors at $10 \mathrm{ng} / \mu \mathrm{L}$ together with pGH8 $10 \mathrm{ng} / \mu \mathrm{L}$, pCFJ90 $2.5 \mathrm{ng} / \mu \mathrm{L}$, pCFJ104 $10 \mathrm{ng} / \mu \mathrm{L}$, pCFJ601 $50 \mathrm{ng} / \mu \mathrm{L}$, and pMA122 $10 \mathrm{ng} / \mu \mathrm{L}$ (gifts from Erik Jorgensen; Addgene $\# 34874$ and \#34873). Injected animals were transferred to new OP50 plates (three per plate) and allowed to generate progeny at $25^{\circ} \mathrm{C}$. One day later, $500 \mu \mathrm{L}$ of $25 \mathrm{mg} / \mathrm{mL}$ G418 was added to each plate for NeoR selection (Thermo Fisher Scientific, USA). Plates were left to starve at $25^{\circ} \mathrm{C}$, and once starved were heatshocked at $34^{\circ} \mathrm{C}$ in an air incubator to kill animals carrying extra-chromosomal arrays. Four hours after heat shock, plates containing animals that were alive, lacked co-injection markers and moved well were chunked onto a fresh OP50 plate and grown at $22{ }^{\circ} \mathrm{C}$. Two days later, single, healthy animals containing successful insertions were selected and placed into individual OP50 plates.

Phenotypic analysis. Axonal breakage was scored in 1-day-old adult animals selected $24 \mathrm{~h}$ prior at the L4 stage in animals containing the $z$ IIs5(Pmec-4::GFP), uIs115(Pmec-17::tagRFP) or $z$ dIs4(Pmec-4::GFP) transgenes. The mean observed proportion of axonal breakage in each strain and $95 \%$ confidence interval was calculated using an Agresti-Coull estimation of binomial distribution.

Laser axotomy. Laser axotomies were performed using a MicroPoint Laser System Basic Unit attached to a Zeiss Axio Imager A1 (Objective EC Plan-Neofluar 100x/ 1.30 Oil M27 $)^{55}$. This laser delivers $120 \mathrm{~mJ}$ of $337 \mathrm{~nm}$ energy with a 2 to $6 \mathrm{~ns}$ pulse length. Axotomies were completed with 10 to 20 pulses in both wild-type and $t b c$ 10(vd31) mutant animals. The PLM axon of animals at the L4 stage was cut at a point $\sim 50 \mu \mathrm{m}$ anterior to the PLM cell body, and the resulting distal fragment was assessed for degeneration $24 \mathrm{~h}$ post-axotomy. Neurons that underwent postaxotomy axonal fusion were excluded from the experiment. The severity of degeneration and clearance was analyzed for each neuron using an arbitrary scale: $1=$ fully cleared axon, $2=$ multiple breaks, $3=$ one break, $4=$ beading and thinning, and $5=$ intact axon $^{56}$.

Microscopy. Animals were inspected by mounting on $4 \%$ agar pads after anesthesia with $0.05 \%$ tetramisole hydrochloride. Fluorescence microscopy was performed using an upright Zeiss AxioImager A1 microscope equipped with a Photometrics Cool Snap HQ2 camera. Images acquired in Metamorph software were further processed in ImageJ v1.51r. Confocal imaging was performed at the Queensland Brain Institute's Advanced Microscopy Facility using a spinning disk confocal microscope (Marianas, Intelligent Imaging Innovations, USA) equipped with a confocal scanner unit (CSU-W1, Yokogawa Electric Co., Japan) built around an Axio Observer body (Z1, Carl Zeiss AG, Germany) and fitted with an sCMOS camera (ORCA-Flash4.0 V2, Hamamatsu Photonics, Japan) and SlideBook v6.0 software (3i, USA) using a $100 \times / 1.46 \mathrm{NA}$ oil-immersion objective with sampling intervals $x, y=63 \mathrm{~nm}$ and $z=130 \mathrm{~nm}$. Images were deconvolved with Huygens Professional v18.04 run on a GPU-accelerated computer (3x NVIDIA ${ }^{\oplus}$ Tesla ${ }^{\circledast}$ V100) using the CMLE algorithm, with signal to noise ratio of 20, background of 100, and 40 iterations. Images exported as 32-bit TIFs and further processed in ImageJ v1.51r to obtain YZ-plane orthogonal views. Microinjections were performed using standard methods ${ }^{57}$, with an inverted Zeiss AxioObserver microscope equipped with differential interference contrast, a Narishige needle holder, and an Eppendorf FemtoJet pump.

Image analysis. Quantification of puncta periodicity and intensity was performed using ImageJ v1.51r and Matlab (Mathworks, USA). Deconvolved confocal $z$-stacks acquired of the PLM neuron, including the neuronal cell body and the first $80-100 \mu \mathrm{m}$ of the axon were converted into maximum image projections of 21 slices centered around the axon. The axon was traced using the ImageJ plugin NeuronJ and an ROI of this trace created for each image. A line scan using this trace as a mask was then obtained and the intensity values for each point extracted. Using Matlab, 793 intensity values for each image were extracted corresponding to a $50 \mu \mathrm{m}$ region of each axon. All intensities were normalized to the mean intensity value observed in wild-type controls at the L4 stage. Autocorrelation curves were generated from these values to measure periodicity. The autocorrelation amplitude was defined as the difference between the first peak and the mean of the two valleys of the autocorrelation curve ${ }^{58}$. Mean puncta spacing was defined as the mean distance between intensity peaks across the region analyzed. Normalized intensities of each genotype at the L4 stage were scored as having gaps in attachment (defined as $>5$ puncta with intensity values $<0.2$ a.u.) or continuous attachment (defined as $<5$ puncta $<0.2$ a.u.). Deconvolved maximum image projections of each genotype at the 1DOA stage were scored for the continuity of VAB-10a::GFP/LET-805:: wrmScarlet along the PLM axon. Localization was scored as continuous (no gaps in localization $>10 \mu \mathrm{m}$ ) or containing gaps (a region of no localization for $>10 \mu \mathrm{m}$ ) with and without axonal breakage. All animals had a large gap in localizations near the anus, which was excluded from the analysis, only the anterior neurite was scored in all examples.

Drug treatments. Animals were grown on NGM agar plates containing $0.1 \mathrm{mM}$ colchicine (Sigma-Aldrich) or $1 \mu \mathrm{M}$ paclitaxel (Sigma-Aldrich) in dimethyl sulfoxide $(\mathrm{DMSO})^{17,59,60}$. For control plates, animals were grown with $1 \%$ DMSO. Parental (P0) animals were grown from the L4 stage on drug plates, and their F1 progeny were scored as 3-day-old adult animals selected at the L4 stage and scored $72 \mathrm{~h}$ later.

RNAi. RNAi was induced by feeding ${ }^{61}$. Fifty milligram per milliliter Amplicillin, 12.5 $\mathrm{mg} / \mathrm{mL}$ Tetracycline, and $1 \mathrm{mM}$ isopropyl $\beta$-D-1-thiogalactopyranoside were added to standard NGM agar, poured into $35 \mathrm{~mm}$ petri dishes and allowed to dry overnight at room temperature. E. coli HT115 carrying either L4440 control empty vector (L4440 was a gift from Andrew Fire; Addgene plasmid \#1654) or unc-70 (clone K11C4.3, Ahringer library ${ }^{62}$ ) were grown in $2 \mathrm{~mL}$ of LB containing $50 \mathrm{mg} / \mathrm{mL}$ Amplicillin and $12.5 \mathrm{mg} / \mathrm{mL}$ Tetracycline at $37^{\circ} \mathrm{C}$ with shaking for $7 \mathrm{~h}$. Several drops of these cultures were then seeded onto the above modified NGM agar and allowed to dry and induce for 2 days. Eight adult animals of each genotype were added to each plate and allowed to lay eggs for $7 \mathrm{~h}$ before being removed. The progeny were then scored for axonal breaks 3 days later as young adults.

Reporting summary. Further information on research design is available in the Nature Research Reporting Summary linked to this article.

\section{Data availability}

The datasets generated and analyzed for Figs. 1, 3, 6, and Supplementary Figs. 2 and 3 are available in the Source Data file. Datasets generated and analyzed for Figs. 4, 5, and 6 and Supplementary Figs. 5 and 6 and all other data are available from the corresponding author on reasonable request.

Received: 8 March 2019; Accepted: 28 November 2019; Published online: 09 January 2020

\section{References}

1. Hilliard, M. A. Axonal degeneration and regeneration: a mechanistic tug-ofwar. J. Neurochem. 108, 23-32 (2009).

2. Gaetz, M. The neurophysiology of brain injury. Clin. Neurophysiol. 115, 4-18 (2004)

3. DeKosky, S. T., Blennow, K., Ikonomovic, M. D. \& Gandy, S. Acute and chronic traumatic encephalopathies: pathogenesis and biomarkers. Nat. Rev. Neurol. 9, 192-200 (2013) 
4. Levy Nogueira, M., Epelbaum, S., Steyaert, J. M., Dubois, B. \& Schwartz, L. Mechanical stress models of Alzheimer's disease pathology. Alzheimers Dement. 12, 324-333 (2016).

5. Wostyn, P., Audenaert, K. \& De Deyn, P. P. Alzheimer's disease and glaucoma: is there a causal relationship? Br. J. Ophthalmol. 93, 1557-1559 (2009).

6. Krieg, M., Dunn, A. R. \& Goodman, M. B. Mechanical control of the sense of touch by beta-spectrin. Nat. Cell Biol. 16, 224-233 (2014).

7. Krieg, M. et al. Genetic defects in beta-spectrin and tau sensitize C. elegans axons to movement-induced damage via torque-tension coupling. Elife 6, https://doi.org/10.7554/eLife.20172 (2017).

8. Ikeda, Y. et al. Spectrin mutations cause spinocerebellar ataxia type 5. Nat. Genet. 38, 184-190 (2006).

9. Hammarlund, M., Jorgensen, E. M. \& Bastiani, M. J. Axons break in animals lacking beta-spectrin. J. Cell Biol. 176, 269-275 (2007).

10. Neumann, B. et al. EFF-1-mediated regenerative axonal fusion requires components of the apoptotic pathway. Nature 517, 219-222 (2015).

11. Emtage, L., Gu, G., Hartwieg, E. \& Chalfie, M. Extracellular proteins organize the mechanosensory channel complex in C. elegans touch receptor neurons. Neuron 44, 795-807 (2004).

12. Vogel, B. E., Muriel, J. M., Dong, C. \& Xu, X. Hemicentins: what have we learned from worms? Cell Res. 16, 872-878 (2006).

13. Pan, C. L., Peng, C. Y., Chen, C. H. \& McIntire, S. Genetic analysis of agedependent defects of the Caenorhabditis elegans touch receptor neurons. Proc. Natl Acad. Sci. USA 108, 9274-9279 (2011).

14. Chaineau, M., Ioannou, M. S. \& McPherson, P. S. Rab35: GEFs, GAPs and effectors. Traffic 14, 1109-1117 (2013).

15. Frasa, M. A., Koessmeier, K. T., Ahmadian, M. R. \& Braga, V. M. Illuminating the functional and structural repertoire of human TBC/RABGAPs. Nat. Rev. Mol. Cell Biol. 13, 67-73 (2012).

16. Patino-Lopez, G. et al. Rab35 and its GAP EPI64C in T cells regulate receptor recycling and immunological synapse formation. J. Biol. Chem. 283, 18323-18330 (2008).

17. Neumann, B. \& Hilliard, M. A. Loss of MEC-17 leads to microtubule instability and axonal degeneration. Cell Rep. 6, 93-103 (2014).

18. Baines, A. J. Evolution of spectrin function in cytoskeletal and membrane networks. Biochem. Soc. Trans. 37, 796-803 (2009).

19. Frokjaer-Jensen, C. Transposon-assisted genetic engineering with Mos1 mediated single-copy insertion (MosSCI). Methods Mol. Biol. 1327, 49-58 (2015).

20. Dickinson, D. J., Pani, A. M., Heppert, J. K., Higgins, C. D. \& Goldstein, B. Streamlined genome engineering with a self-excising drug selection cassette. Genetics 200, 1035-1049 (2015).

21. Stenmark, H. Rab GTPases as coordinators of vesicle traffic. Nat. Rev. Mol. Cell Biol. 10, 513-525 (2009).

22. Haley, R., Wang, Y. \& Zhou, Z. The small GTPase RAB-35 defines a third pathway that is required for the recognition and degradation of apoptotic cells. PLoS Genet. 14, e1007558 (2018).

23. Kutscher, L. M., Keil, W. \& Shaham, S. RAB-35 and ARF-6 GTPases mediate engulfment and clearance following linker cell-type death. Dev. Cell 47, 222-238 (2018). e226.

24. Sato, M. et al. Regulation of endocytic recycling by C. elegans Rab35 and its regulator RME-4, a coated-pit protein. EMBO J. 27, 1183-1196 (2008).

25. Francis, R. \& Waterston, R. H. Muscle cell attachment in Caenorhabditis elegans. J. Cell Biol. 114, 465-479 (1991).

26. Chalfie, M. \& Sulston, J. Developmental genetics of the mechanosensory neurons of Caenorhabditis elegans. Dev. Biol. 82, 358-370 (1981).

27. Hresko, M. C., Schriefer, L. A., Shrimankar, P. \& Waterston, R. H. Myotactin, a novel hypodermal protein involved in muscle-cell adhesion in Caenorhabditis elegans. J. Cell Biol. 146, 659-672 (1999).

28. Woo, W. M., Goncharov, A., Jin, Y. \& Chisholm, A. D. Intermediate filaments are required for C. elegans epidermal elongation. Dev. Biol. 267, 216-229 (2004).

29. Bosher, J. M. et al. The Caenorhabditis elegans vab-10 spectraplakin isoforms protect the epidermis against internal and external forces. J. Cell Biol. 161, 757-768 (2003).

30. Yang, Y. et al. Spectraplakin induces positive feedback between fusogens and the actin cytoskeleton to promote cell-cell fusion. Dev. Cell 41, 107-120 (2017). e104.

31. Labouesse, M. Epithelial junctions and attachments. WormBook, 1-21, https:// doi.org/10.1895/wormbook.1.56.1 (2006).

32. Zhang, H. et al. A tension-induced mechanotransduction pathway promotes epithelial morphogenesis. Nature 471, 99-103 (2011).

33. Hu, S., Pawson, T. \& Steven, R. M. UNC-73/trio RhoGEF-2 activity modulates Caenorhabditis elegans motility through changes in neurotransmitter signaling upstream of the GSA-1/Galphas pathway. Genetics 189, 137-151 (2011).

34. Raizen, D. M. et al. Lethargus is a Caenorhabditis elegans sleep-like state. Nature 451, 569-572 (2008).
35. Machnicka, B. et al. Spectrins: a structural platform for stabilization and activation of membrane channels, receptors and transporters. Biochim. Biophys. Acta 1838, 620-634 (2014).

36. Leshchyns'ka, I., Sytnyk, V., Morrow, J. S. \& Schachner, M. Neural cell adhesion molecule (NCAM) association with PKCbeta2 via betaI spectrin is implicated in NCAM-mediated neurite outgrowth. J. Cell Biol. 161, 625-639 (2003).

37. Leshchyns'ka, I., Tanaka, M. M., Schachner, M. \& Sytnyk, V. Immobilized pool of NCAM180 in the postsynaptic membrane is homeostatically replenished by the flux of NCAM180 from extrasynaptic regions. J. Biol. Chem. 286, 23397-23406 (2011).

38. Tian, N. et al. Lipid raft-dependent endocytosis of close homolog of adhesion molecule L1 (CHL1) promotes neuritogenesis. J. Biol. Chem. 287, 44447-44463 (2012).

39. Dubreuil, R. R., Wang, P., Dahl, S., Lee, J. \& Goldstein, L. S. Drosophila beta spectrin functions independently of alpha spectrin to polarize the $\mathrm{Na}, \mathrm{K}$ ATPase in epithelial cells. J. Cell Biol. 149, 647-656 (2000).

40. Barres, B. A. The mystery and magic of glia: a perspective on their roles in health and disease. Neuron 60, 430-440 (2008).

41. Susuki, K. et al. Glial betaII spectrin contributes to paranode formation and maintenance. J Neurosci., https://doi.org/10.1523/JNEUROSCI.3647-17.2018 (2018).

42. Susuki, K. et al. Schwann cell spectrins modulate peripheral nerve myelination. Proc. Natl Acad. Sci. USA 108, 8009-8014 (2011).

43. Garcia-Fresco, G. P. et al. Disruption of axo-glial junctions causes cytoskeletal disorganization and degeneration of Purkinje neuron axons. Proc. Natl Acad. Sci. USA 103, 5137-5142 (2006)

44. Ritchie, F. K. et al. The heterochronic gene lin-14 controls axonal degeneration in C. elegans neurons. Cell Rep. 20, 2955-2965 (2017).

45. Vogel, B. E. \& Hedgecock, E. M. Hemicentin, a conserved extracellular member of the immunoglobulin superfamily, organizes epithelial and other cell attachments into oriented line-shaped junctions. Development 128, 883-894 (2001).

46. Zahreddine, H., Zhang, H., Diogon, M., Nagamatsu, Y. \& Labouesse, M. CRT1/calreticulin and the E3 ligase EEL-1/HUWE1 control hemidesmosome maturation in C. elegans development. Curr. Biol. 20, 322-327 (2010).

47. Osmani, N. et al. An Arf6- and caveolae-dependent pathway links hemidesmosome remodeling and mechanoresponse. Mol. Biol. Cell 29, 435-451 (2018).

48. Brenner, S. The genetics of Caenorhabditis elegans. Genetics 77, 71-94 (1974).

49. Minevich, G., Park, D. S., Blankenberg, D., Poole, R. J. \& Hobert, O. CloudMap: a cloud-based pipeline for analysis of mutant genome sequences. Genetics 192, 1249-1269 (2012).

50. Afgan, E. et al. The Galaxy platform for accessible, reproducible and collaborative biomedical analyses: 2018 update. Nucl. Acids Res. 46, W537-W544 (2018).

51. Zuryn, S., Le Gras, S., Jamet, K. \& Jarriault, S. A strategy for direct mapping and identification of mutations by whole-genome sequencing. Genetics 186, 427-430 (2010).

52. Sambrook, J., Fritsch, E. F. \& Maniatis, T. Molecular Cloning: A Laboratory Manual. (Cold Spring Harbour Laboratory Press, 1989).

53. Gibson, D. G. Enzymatic assembly of overlapping DNA fragments. Methods Enzymol. 498, 349-361 (2011).

54. Gibson, D. G. et al. Enzymatic assembly of DNA molecules up to several hundred kilobases. Nat. Methods 6, 343-345 (2009).

55. Neumann, B., Nguyen, K. C., Hall, D. H., Ben-Yakar, A. \& Hilliard, M. A. Axonal regeneration proceeds through specific axonal fusion in transected $C$ elegans neurons. Dev. Dyn. 240, 1365-1372 (2011).

56. Nichols, A. L. A. et al. The apoptotic engulfment machinery regulates axonal degeneration in C. elegans neurons. Cell Rep. 14, 1673-1683 (2016).

57. Mello, C. C., Kramer, J. M., Stinchcomb, D. \& Ambros, V. Efficient gene transfer in C. elegans: extrachromosomal maintenance and integration of transforming sequences. EMBO J. 10, 3959-3970 (1991).

58. He, J. et al. Prevalent presence of periodic actin-spectrin-based membrane skeleton in a broad range of neuronal cell types and animal species. Proc. Natl Acad. Sci. USA 113, 6029-6034 (2016).

59. Chalfie, M. \& Thomson, J. N. Structural and functional diversity in the neuronal microtubules of Caenorhabditis elegans. J. Cell Biol. 93, 15-23 (1982).

60. Kirszenblat, L., Neumann, B., Coakley, S. \& Hilliard, M. A. A dominant mutation in mec-7/beta-tubulin affects axon development and regeneration in Caenorhabditis elegans neurons. Mol. Biol. Cell 24, 285-296 (2013).

61. Timmons, L., Court, D. L. \& Fire, A. Ingestion of bacterially expressed dsRNAs can produce specific and potent genetic interference in Caenorhabditis elegans. Gene 263, 103-112 (2001).

62. Kamath, R. S. \& Ahringer, J. Genome-wide RNAi screening in Caenorhabditis elegans. Methods 30, 313-321 (2003). 


\section{Acknowledgements}

We thank L. Hammond, R. Amor, A. Thompson, and A. Gaudin for support with microscopy; J. Carroll and I. Porebski for IT support; J. Edson, I. Makunin, G. Minevich, and O. Hobert for technical assistance with Whole-Genome Sequencing; A. Ho for help with statistical analysis; J. Götz, S. Millard, F. Meunier, K. Shen, B. Neumann, and R. Tweedale, for comments on the manuscript; S. Zuryn, Z. Hu, Z. Li, and members of the Zuryn, $\mathrm{Hu}, \mathrm{Li}$, and Hilliard laboratories for discussions and comments; and M. Chalfie, M. Hammarlund, E. Jorgensen, M. Goodman, B. Grant, B. Goldstein, $\mathrm{K}$. Shen, and S. Zuryn for sharing reagents. Some strains were provided by the CGC, which is funded by NIH Office of Research Infrastructure Programs (P40 OD010440), and the International C. elegans Gene Knockout Consortium. This work was supported by NHMRC Project Grant 1145896 and NHMRC Senior Research Fellowship 1111042 to M.A.H.; NHMRC-ARC Dementia Research Development Fellowship Grant 1108489 to S.C.

\section{Author contributions}

S.C., F.K.R., and K.M.G. carried out all experiments. S.C. and M.A.H. designed and interpreted experiments, and wrote the paper.

\section{Competing interests}

The authors declare no competing interests.

\section{Additional information}

Supplementary information is available for this paper at https://doi.org/10.1038/s41467019-13795-x.
Correspondence and requests for materials should be addressed to M.A.H.

Reprints and permission information is available at http://www.nature.com/reprints

Peer Review Information Nature Communications thanks Jon Morrow and Shuzo Sugita for their contribution to the peer review of this work.

Publisher's note Springer Nature remains neutral with regard to jurisdictional claims in published maps and institutional affiliations.

\begin{abstract}
(c) (i) Open Access This article is licensed under a Creative Commons Attribution 4.0 International License, which permits use, sharing, adaptation, distribution and reproduction in any medium or format, as long as you give appropriate credit to the original author(s) and the source, provide a link to the Creative Commons license, and indicate if changes were made. The images or other third party material in this article are included in the article's Creative Commons license, unless indicated otherwise in a credit line to the material. If material is not included in the article's Creative Commons license and your intended use is not permitted by statutory regulation or exceeds the permitted use, you will need to obtain permission directly from the copyright holder. To view a copy of this license, visit http://creativecommons.org/ licenses/by/4.0/.
\end{abstract}

(C) The Author(s) 2020 